\title{
Variación del clima de un Terroir y su consecuencia sobre la respuesta de la vid
}

\section{Variation of the climate of a Terroir and its consequence on the vine's response}

\author{
Milka Ferrer $^{1, \mathrm{a}}$, Gerardo Echeverría ${ }^{1}$, Gustavo Pereyra ${ }^{1}$, Julia Salvarrey ${ }^{1}$, Leandro Arrillaga ${ }^{1}$ y Mercedes Fourment ${ }^{1}$ \\ ${ }^{1}$ Departamento de Producción Vegetal, Facultad de Agronomía, Av. Garzón 780 Montevideo,Uruguay \\ a'mferrer@fagro.edu.uy
}

\begin{abstract}
Resumen
El clima vitícola, un componente mayor del Terroir, fue identificado en la región sur del Uruguay: sequía moderada, templado cálido, noches templadas, sometido a la influencia de la brisa marina. En el período 19942016, se analizó el régimen térmico e hídrico y su influencia sobre el rendimiento, sanidad y composición de la uva, de viñedos comerciales de Tannat. ACP mostró 3 grupos de años: Grupo 1 (húmedo), precipitaciones del ciclo del cultivo superiores a la media, sanidad limitante, acidez y rendimiento mayores a la media y azúcares inferiores, cosechas tardías. Grupo 2 (cálido y seco), condiciones térmicas superiores al promedio y la componente hídrica por debajo, sanidad superior a la media, azúcares y acidez inferiores, cosechas adelantadas. Grupo 3 (fresco, húmedo en primavera y seco en maduración), condiciones térmicas inferiores a la media, precipitaciones superiores a la media en brotación -cuajado e inferiores en el mes previo a cosecha, superiores a la media azúcares y tamaño de baya, cosecha atrasada. Se establecieron correlaciones entre las variables del clima, rendimiento y la calidad de la uva. En la serie de años estudiada, la variabilidad climática de la región fue muy marcada y la respuesta de Tannat acompañó dicha variabilidad.
\end{abstract}

Palabras clave: Variabilidad climática, rendimiento, sanidad, composición de la uva, Tannat

\begin{abstract}
Summary
Viticultural climate, a major component of Terroir, in Uruguay southern region was classified as moderately dry, temperate-warm, with temperate nights and influenced by sea breeze. During the 1994-2016 period, thermal and water regimes and their influence on grapevine yield, sanitary status and berry composition was analyzed for Tannat cultivar grown in commercial vineyards. Principal component analysis (PCA) separated years in three groups: Group 1 (wet), rainfall over the growing season higher than the average, sanitary status is limiting, acidity and yield higher than the average, whereas sugar content is lower, late harvest. Group 2 (warm and dry), thermal conditions greater and water component lower than the average, better sanitary status, sugar contents and acidity lower than the average, early harvest. Group 3 (fresh, wet in spring and dry during ripening), thermal conditions lower than the average, rainfall higher during budbreak-fruitset and lower than the average in the month previous to harvest, berry size and sugar contents greater than the average, late ripening. Correlations among climate, yield and berry quality variables were established. In the studied years, climate variability within the region was high and the response of the Tannat cultivar was in accordance to this variability.
\end{abstract}

Keywords: climate variability, yield, sanitary status, berry composition, Tannat

\section{Introducción}

El clima es uno de los componentes mayores del Terroir. El clima vitícola de la región sur del Uruguay fue identificado como de sequía moderada, templado cálido, noches templadas (CCM) [1], sometido a la influencia de la brisa marina delestuario del Rio de la Plata [2, 3]. En el contexto de cambio climático, para el Uruguay se prevé una mayor variabilidad interanual de las precipitaciones, en particular durante el período de maduración de la uva, así como un aumento de la temperatura media [4]. Esta situación condiciona el rendimiento y la composición de la uva y favorece la aparición de enfermedades fúngicas que determinan pudriciones a nivel de racimos ocasionando distorsión en la producción y en la calidad de la uva $[5,6]$.

Las variaciones que se registran en el rendimiento dependen principalmente del número de racimos que se indujeron en la temporada anterior. Este proceso 
fisiológico dentro de la yema, ocurre durante el período de floración de la temporada en curso, por lo que es necesario un buen aporte de las reservas y cuya movilización, depende fundamentalmente de un aporte hídrico no limitante $[7,8]$. Uno de los componentes del rendimiento mayormente afectado por la temperatura y por disponibilidad hídrica es la baya. Condiciones térmicas durante la maduración o déficit hídricos en la primera etapa de su desarrollo determinan fuertemente su tamaño [9-12].

Las condiciones meteorológicas explican más del $80 \%$ de la variabilidad en la composición de la uva [13]. La temperatura, en particular durante el período de maduración, tiene una incidencia directa sobre la degradación de los ácidos orgánicos. Rangos de temperaturas superiores a los $25-30{ }^{\circ} \mathrm{C}$ pueden ser limitantes para la fotosíntesis y por lo tanto para la acumulación de azúcares en la baya $[11,14]$. Las precipitaciones según la etapa del ciclo del cultivo tienen un efecto negativo o positivo en el contenido de azúcares y de los ácidos [13,14].

En nuestro ambiente, Botrytis cinerea es de las enfermedades que determinan mayores pérdidas en rendimiento y calidad de la uva $[12,15]$. Los factores climáticos predisponentes para la infección de este hongo, son las precipitaciones abundantes durante la maduración y las condiciones térmicas templadas $[12,16]$.

En nuestros diferentes terroirs, la variedad Tannat, considerada insignia del país, aporta vinos con gran tipicidad y originalidad y expresa a su gran potencial enológico [17].

El objetivo de este trabajo fue analizar en un contexto de un clima cambiante y en función de las condiciones agro-meteorológicas de un período de 22 años (1994-2016), la variación del régimen térmico e hídrico y su influencia sobre el rendimiento, sanidad y composición de la uva variedad Tannat.

\section{Materiales y Métodos}

\subsection{Ubicación del ensayo}

La información de campo se obtuvo de tres parcelas de viñedos comerciales de la variedad Tannat injertada sobre SO4, conducidas en espaldera alta y con poda Guyot, con filas orientadas N-S, ubicadas en el Departamento de Canelones (Lat.:34 $37^{\prime} \mathrm{S} ; 56^{\circ} 17^{\prime} \mathrm{W}$ ). El clima vitícola de esta región fue calificado como de sequía moderada, templado cálido, con noches templadas, según la "Clasificación Climática Multicriterios" [1,18]. La información del cultivo provino de proyectos de investigación del grupo de Viticultura de la Facultadde Agronomía, correspondientes al periodo 1994 a 2016, siguiendo protocolos específicos para cada variable.

La información de las variables climáticas se tomó de la base de datos de la estación meteorológica del Instituto Nacional de Investigación Agropecuaria (INIA)

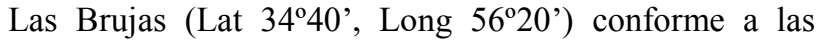
normas de la OMM. La estación está situada en laregión de estudio y abarca un radio de $14 \mathrm{~km}$. Las variables climáticas diarias consideradas se presentan en la Tabla 1. Se calculó los Grados Días base 10 y el número de días desde el $1^{\circ}$ de Enero hasta la fecha de cosecha de cada año. La escala temporal fue de 23 años (1994 a 2016 inclusive), correspondientes a la misma serie de registros del cultivo.

\subsection{Evaluaciones sobre la planta de cada parcela}

Componentes del rendimiento $(\mathrm{kg} / \mathrm{ha})$ : en cosecha, en 30 plantas se pesó individualmente: uva sana y enferma y se calculó el porcentaje del total. El peso de baya (g) (balanza Ohaus Scout, Ohaus Corp., USA) se obtuvo sobre una muestra de 250 bayas por duplicado tomadas según protocolo propuesto por Carbonneau [19]. Para la determinación del estado sanitario (enfermo) se tuvo en cuenta los racimos con más de $5 \%$ de incidencia de podredumbres de racimo.

Tabla 1: Variables Climáticas

\begin{tabular}{|l|l|}
\hline Variable & Sigla \\
\hline $\begin{array}{l}\text { Temperatura Max Enero } \\
\left({ }^{\circ} \mathrm{C}\right)\end{array}$ & $\mathrm{TME}$ \\
\hline $\begin{array}{l}\text { Temperatura Max Febrero } \\
\left({ }^{\circ} \mathrm{C}\right)\end{array}$ & $\mathrm{TMF}$ \\
\hline $\begin{array}{l}\text { Temperatura min Febrero } \\
\left({ }^{\circ} \mathrm{C}\right)\end{array}$ & $\mathrm{TmF}$ \\
\hline $\begin{array}{l}\text { Grado Días (Enero cosecha, } \\
\left.{ }^{\circ} \mathrm{C}\right)\end{array}$ & $\mathrm{GDD}$ \\
\hline $\begin{array}{l}\text { Grado Días Diario (Enero } \\
\left.\text { cosecha, }{ }^{\circ} \mathrm{C}\right)\end{array}$ & $\mathrm{GDDD}$ \\
\hline $\begin{array}{l}\text { Número de días T }>\text { a } 30{ }^{\circ} \mathrm{C} \\
(1 \mathrm{Noviembre}-\text { cosecha) }\end{array}$ & $\mathrm{N}{ }^{\circ} \mathrm{D}>30^{\circ} \mathrm{C} \mathrm{N}-\mathrm{co}$ \\
\hline $\begin{array}{l}\text { Precipitaciones Setiembre }- \\
\text { Octubre (mm) }\end{array}$ & $\mathrm{PP} \mathrm{St-Oc}$ \\
\hline $\begin{array}{l}\text { Precipitaciones Noviembre }- \\
\text { Diciembre (mm) }\end{array}$ & $\mathrm{PP}$ Nv-Dc \\
\hline $\begin{array}{l}\text { Precipitaciones Enero - } \\
\text { Cosecha (mm) }\end{array}$ & $\mathrm{PP}$ En-Cs \\
\hline $\begin{array}{l}\text { Precipitaciones Ciclo del } \\
\text { cultivo (mm) }\end{array}$ & $\mathrm{PPCiclo}$ \\
\hline $\begin{array}{l}\text { Precipitaciones del mes } \\
\text { previo a cosecha (mm) }\end{array}$ & $\mathrm{PP} 1 \mathrm{MC}$ \\
\hline $\begin{array}{l}\text { Número de días a cosecha } \\
(1 \text { Enero - cosecha) }\end{array}$ & $\mathrm{N}{ }^{\circ} \mathrm{dC}(1 \mathrm{En}-\mathrm{co})$ \\
\hline
\end{tabular}

Composición de la uva: en la cosecha, sobre las muestras de 250 bayas en las que se midió el peso y a partir del método de la O.I.V [20], se determinó: sólidos solubles por refractometría (Atago, Master-T, Japón) y acidez total (AT), por titulación (Bureta Mohr).Para el contenido de antocianos, se tomaron tres muestras de 250 bayas para determinar el potencial polifenólico (potencial total en antocianos ApH 1,0, (se presentan datos de 3 años), siguiendo la metodología propuesta por Glories y Augustin [21] modificado por González-Neves [22].

Análisis estadístico de los resultados: éstos fueron analizados utilizando las técnicas estadísticas multivariadas de análisis de componentes principales (ACP) para separar las variables asociadas yel coeficiente 


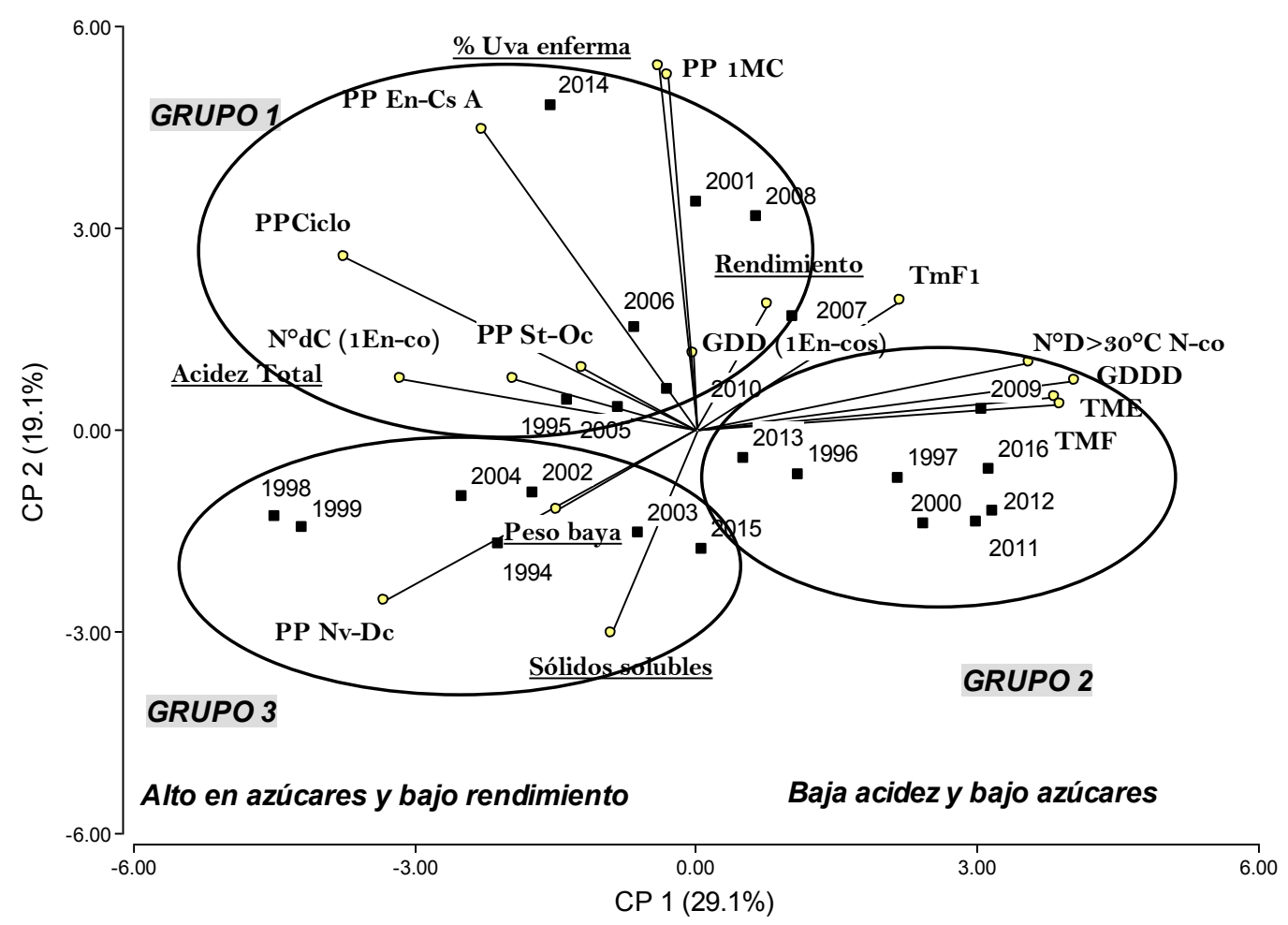

Figura 1. Análisis de Componentes Principales: Años, factores del clima y respuesta de la planta. Abreviaciones listadas en la Tabla 1

de correlación de Pearson para evaluar las posibles relaciones entre las diferentes variables consideradas. Los análisis se llevaron a cabo empleando el software InfoStat [23].

\section{Resultados y discusión}

\subsection{Grupo de años}

En el análisis de ACP, los dos primeros componentes principales explican el 48,2\% de la varianza total (Figura 1). El componente principal 1 (CP1) aporta el 29,1\% mientras que el componente principal 2 (CP2) aporta el 19,1\%. Los vectores de carga que integran el ACP, se encuentran asociados entre sí en distinta magnitud formando 3 grupos de años que se caracterizaron en función de las medias de las variables del período analizado y contribuyendo de manera diferente a cada CP.

Los grupos comprenden prácticamente el mismo número de años $(8,8$ y 7$)$, pero con condiciones meteorológicas contrastantes, que condicionan principalmente la composición de la uva y su sanidad, en acuerdo con van Leeuwen et al. [13].

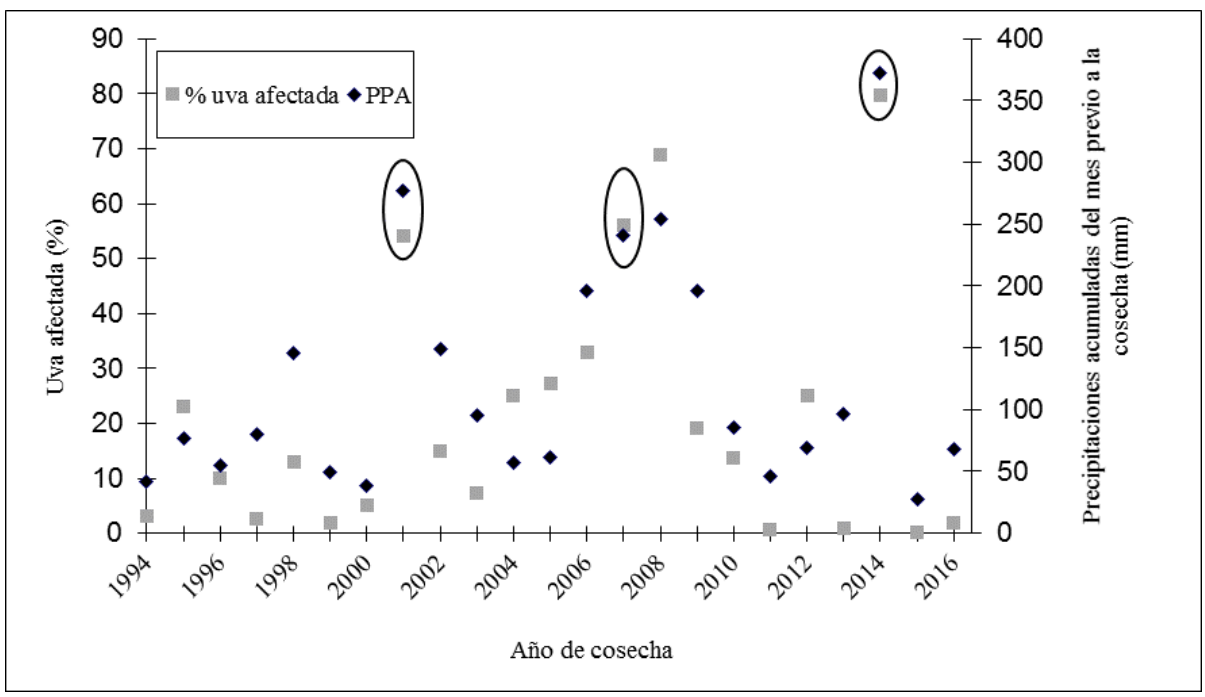

Figura 2. Relación entre las precipitaciones del mes previo a la cosecha y el Porcentaje de uva enferma 
El Grupo 1 (húmedo) comprende 8 años, asociado a precipitaciones superiores a la media durante todo el ciclo del cultivo, así como las temperaturas nocturnas del mes previo a la cosecha. Estas condiciones generaron que el estado sanitario fuera limitante, el rendimiento y la acidez del mosto fueran superiores a la media, en tanto que por debajo del promedio estuvieron los azúcares y se constató una fecha de cosecha tardía. Estos resultados, en función de las condiciones meteorológicas, son citados por numerosos autores $[7,8$, 12-14, 16]. La mayor incidencia de podredumbres de racimo se correlacionó positiva y significativamente con las precipitaciones del mes anterior a la cosecha (PP1MC, $\mathrm{r}=0,88, \mathrm{p}<0,0001$ ), con las del período de maduración (PP En-Cs, $r=0,68, p=0,0004)$ y con las del ciclo del cultivo (PP Ciclo, $r=0,42 . p=0,04)$. En la figura 2 se muestra la correlación entre las precipitaciones del mes previo a la cosecha y el porcentaje de uva enferma por pudriciones de racimos. Otra consecuencia de las precipitaciones es el aumento del vigor de las plantas que está asociado a mayores rendimientos y efectos sobre la composición de la uva $[24,25]$. En este sentido, las precipitaciones que se correlacionaron positivamente con la acidez, son las del ciclo del cultivo (PPciclo, $r=0,46, p=0,03$ ), las de maduración ( $\mathrm{PP}$ En-Cs, $\mathrm{r}=0,34, \mathrm{p}=0,1$ ) y las del mes previo a la cosecha, negativamente con la cantidad de azúcares en la baya $(\mathrm{r}=-0,42, \mathrm{p}=0,04)$. Este último resultado, probablemente se asocie al efecto de dilución en esta etapa [25]. Las temperaturas también juegan un rol sobre la acidez [26]. Las temperaturas durante la maduración (TME y TMF) estuvieron en valores inferiores a la media del período analizado $\left(29\right.$ y $28{ }^{\circ} \mathrm{C}$ respectivamente), a la vez que éstas se correlacionaron negativa y significativamente con la acidez (TME, r=0,53, $\mathrm{p}=0,0092, \mathrm{TMF}, \mathrm{r}=-0,37, \mathrm{p}=0,08)$. Esta degradación más lenta, en particular del ácido málico, prolongó el período de maduración $(\mathrm{r}=0,61, \mathrm{p}=0,002)$, en la medida que la acidez total fue uno de los índices de cosecha considerado (Figura 3).

En el Grupo 2 (cálido y seco), que comprende 8 años, las condiciones térmicas estuvieron por encima del

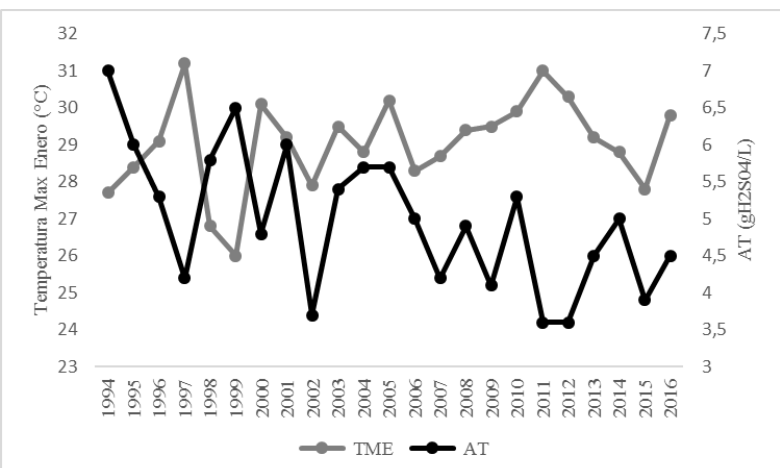

Figura 3. Relación entre la acidez total y la temperatura máxima del mes más cálido

promedio y la componente hídrica por debajo de lo esperado durante todo el ciclo del cultivo. Estas condiciones tuvieron como consecuencia un buen estado sanitario, en tanto que azúcares y acidez fueron inferiores a la media y como era de esperar con cosechas adelantadas. Los bajos contenidos de acidez se corresponden, como ya fue citado, a su degradación como consecuencia de las altas temperaturas durante la maduración. Por otra parte, también influyeron negativamente en el proceso de fotosíntesis. Como es comunicado por varios autores, en rangos de temperaturas superiores a los 25-30 ${ }^{\circ} \mathrm{C}$ puede ser limitante para la fotosíntesis y por lo tanto para la acumulación de azúcares en la baya $[11,14]$. En nuestro estudio, la mayoría de los años de este grupo la temperatura máxima superó los $30{ }^{\circ} \mathrm{C}$. Condiciones de estrés hídrico son reportadas en la bibliografía como causa del bloqueo de la fotosíntesis [28]. En el caso de nuestro trabajo, las precipitaciones registradas durante el ciclo del cultivo, cuando se las compara con la media de los años, en 7 de los 8 años estuvo por debajo de esta media $(643 \mathrm{~mm})$, con extremos de $-131 \mathrm{~mm}$ a $-333 \mathrm{~mm}$. El balance hídrico del 2016, según los valores propuestos [1], se catalogó de Índice de sequía fuerte $(-177 \mathrm{~mm})$ cuando el clima de la región lo cataloga de Sequía moderada con valores históricos del Índice de $50 \mathrm{~mm}$.

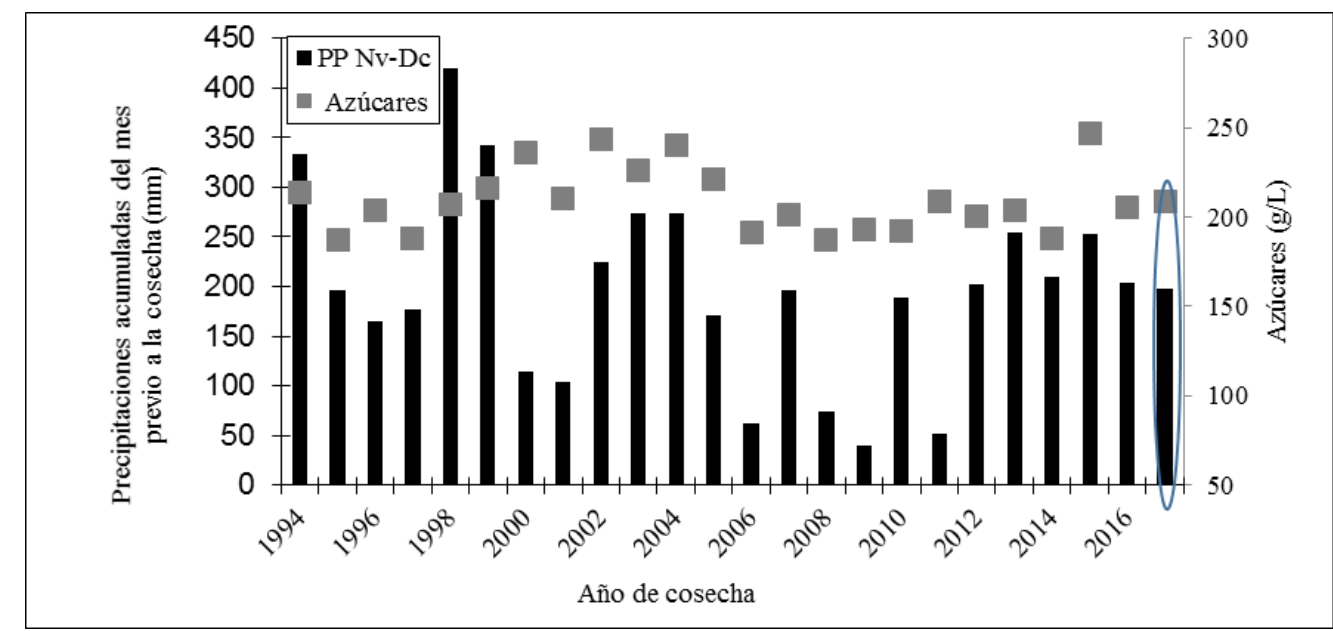

Figura 4. Relación entre las Precipitaciones del mes previo a la cosecha y el contenido de azúcares en la baya 
Tabla 2. Año representativo de cada grupo

\begin{tabular}{|c|c|c|c|}
\hline & $\begin{array}{c}\text { Grupo 1 (cálido en } \\
\text { maduración y húmedo) } \\
\text { Año 2014 }\end{array}$ & $\begin{array}{c}\text { Grupo 2 (cálido y seco) } \\
\text { Año 2016 }\end{array}$ & $\begin{array}{c}\text { Grupo 3 (fresco, } \\
\text { húmedo en primavera y } \\
\text { seco en maduración) } \\
\text { Año 2002 }\end{array}$ \\
\hline $\begin{array}{c}\text { Acidez Total } \\
\text { (gH2SO4/L) }\end{array}$ & 5,0 & 3,9 & 4,1 \\
\hline Azúcares (g/L) & 188 & 205 & 243 \\
\hline ApH1 & 784 & 1756 & 3632 \\
\hline Rendimiento (kg/ha) & 23430 & 22140 & 16900 \\
\hline Peso de la baya (g) & 1,53 & 1,31 & 1,62 \\
\hline Uva enferma (\%) & 79,8 & 0,75 & 1,5 \\
\hline
\end{tabular}

El Grupo 3 (fresco, húmedo en primavera y seco en maduración), comprende 7 años, asociado a condiciones térmicas por debajo de la media, temperaturas nocturnas bajas en el mes previo a la cosecha, y las precipitaciones fueron superiores a la media en el período brotación -cuajado e inferiores en el mes previo a cosecha. Estas condiciones favorecieron el mayor contenido de azúcares así como el tamaño de baya, los que estuvieron por encima de la media. El rendimiento por hectárea fue inferior a la media, la cosecha en estas condiciones fue atrasada y no se registraron problemas sanitarios. El componente hídrico de período floración - cuajado, que se correlacionó positivamente con el contenido de azúcares (PP Nv.Dc, $\mathrm{r}=0,48, \mathrm{p}=0,04)$, ya que permitió una buena instalación de la superficie foliar, que favoreció la producción de fotosintatos, e influyó positivamente en el tamaño de la baya en acuerdo con lo comunicado por varios autores [9, 29], (Figura 4). El tamaño de la baya fue afectado negativamente por las temperaturas elevadas durante la maduración $\left(\mathrm{TMF}, \mathrm{r}=-0,42, \mathrm{p}=0,04, \mathrm{~N}^{\circ} \mathrm{D}>30^{\circ} \mathrm{C} \mathrm{N}\right.$-co $\mathrm{r}=$ $0,46, \mathrm{p}=0,02)$. En este grupo de años, las temperaturas TMF y N ${ }^{\circ} \mathrm{D}>30^{\circ} \mathrm{C}$ estuvieron por debajo de la media, por lo que no causaron su deshidratación en la etapa final de la maduración de la baya [10]. Del punto de vista enológico este grupo de años fue el que se destacó por la calidad de sus vinos.

En la Tabla 2 se muestra el rendimiento, sanidad y la composición de la uva de un año representativo de cada grupo.

\section{Conclusiones}

Para la serie de años estudiada, la variabilidad climática de la región fue muy marcada $\mathrm{y}$, en consecuencia, se observó una respuesta de Tannat acorde a dicha variabilidad. Esto muestra la importancia que tiene el clima para nuestras condiciones de cultivo, por lo que para evaluar el potencial del terroir vitícola del sur de Uruguay, se deben continuar los estudios del rol de los diferentes componentes del clima. Las condiciones meteorológicas del año tienen marcada influencia en la calidad enológica de la uva, siendo las del grupo 2 las más favorables.

\section{Referencias}

1. J. Tonietto y A. Carbonneau Agric. Forest Meteorol. 124, 81-97 (2004)

2. M. Fourment, M. Ferrer, G. González-Neves, G. Barbeau, V. Bonnardot, H. Quénol, Int. J. Biomet. 61, 1617-1628 (2017)

3. G. Echeverría, Tesis doctoral. Montevideo Uruguay, Facultad de Agronomía, Universidad de la República. 233p (2017)

4. G. Tiscornia; A. Cal; A. Gimenez Rev Invest. Agrop RIA, 42 1, p.: 66 - 71 ( 2016)

5. R. Mira de Orduña, Food Res. Inter., 43, 1844 - 855 (2010)

6. M. Ferrer, G. Gonzalez-Neves; G. Echeverría, A. Carbonneau, Progrès Agric. Vitic. 128, 367-371 (2011)

7. N. Guilpart, A. Metay, CH. Gary. Eur. J. Agron. 54, 9-20 (2014)

8. M. Mendez-Costabel, K. Wilkinson, S. Bastian, C. Jordans, M. McCarthy, C. Ford, N. Dokoozlian, Aust. J. Grape Wine Res. 20, 100-110 (2014)

9. H. Ojeda, C. Andary, E. Kraeva, A. Carbonneau, A. Deloire, Am. J. Enol. Vitic. 53 4, 261 - 267 (2002)

10. S. Rogiers, J. Hatflied, V. Guntajaudzems, R. White, M. Keller. Am. J. Enol. Vitic. 55, 121-127 (2004)

11. M. Ferrer, G. Echeverria, A. Carbonneau, S. Afr. J. Enol. Vitic. 35, 103-113 (2014)

12. M. Ferrer, G. Echeverría, J.M. Miras-Avalos, Inter. J. Env. Agric. Res. 3, 16-27 (2017)

13. C. van Leeuwen, P. Friant, X. Choné, O. Tregoat, S. Koundouras, D. Dubourdieu, Am. J. Enol. Vitic. 55, 207-217 (2004)

14. J. Hunter, V. Bonnardot, S. Afr. J. Enol. Vitic. 32, $137-$ $154(2011)$

15. V. Gepp, S. Vero, M. Cassanello, G. Romero, E. Silvera, P. González, J. Rebellato, Y. Ferreira, O. Bentancur, Agrocien. 16, 97-107 (2012)

16. E. González-Domínguez, T. Caffi, N. Ciliberti, V. Rossi, PlosOne 10 (2015)

17. G. González-Neves, G. Gil, L. Barreiro, M. Ferrer, J. Franco, Agrocien. 10, 1-14 (2006)

18. M. Ferrer, Tesis doctoral. Montpellier, Francia. Université Montpellier II, 388p (2007) 
19. A. Carbonneau, A. Moueix, N. Leclair, J. Renoux, Bull. OIV 727, 679-690 (1991)

20. O.I.V. Office Internationale de la Vigne et du vin. Paris, France, 388p (2009)

21. Y. Glories; M. Agustin, Proc. Journée Technique CIVB, Bordeaux, France pp 56-61 (1993

22. G. González-Neves, Tesis Doctoral, Montpellier, Francia. Université de Montpellier II. 279p (2005)

23. J.A. di Rienzo, F. Casanoves, M.G. Balzarini, L. González, M. Tablada, C.W. Reboledo, Infostat versión 2012, Universidad Nacional de Córdoba (2013)
24. I. Filippetti, G. Allegro, G. Valentini, C. Pastore, E. Colucci, C. Intrieri, J. Int. Sci. Vigne Vin, 47, 21-33 (2013)

25. M.C. Ramos, J.A. Martínez-Casanovas, Plant Soil 333, $375-$ $389(2010)$

26. R.R. Walker, D.H. Blackmore, P.R.Clingeleffer, G.H. Kerridge, E.H. Ruth, P.R. Nicholas, Aust. J. Grape Wine Res, 11, 2-8 (2005).

27. M. Keller, Aust. J. Grape Wine Res. 16, 56-69 (2010)

28. J. Bota, O. Stayk, J. Flexas, H. Medrano, Func. Plant Biol. 31, 697-708 (2004)

29. P. Costenza, B Tisseyre, JJ. Hunter, A. Deloire, S. Afr. J. Enol. 25, 43-47 (2004) 